TRANSACTIONS OF THE

AMERICAN MATHEMATICAL SOCIETY

Volume 363, Number 12, December 2011, Pages 6531-6548

S 0002-9947(2011)05345-6

Article electronically published on July 25, 2011

\title{
UNIVERSAL VECTOR BUNDLE OVER THE REALS
}

\author{
INDRANIL BISWAS AND JACQUES HURTUBISE
}

\begin{abstract}
Let $X_{\mathbb{R}}$ be a geometrically irreducible smooth projective curve, defined over $\mathbb{R}$, such that $X_{\mathbb{R}}$ does not have any real points. Let $X=X_{\mathbb{R}} \times_{\mathbb{R}} \mathbb{C}$ be the complex curve. We show that there is a universal real algebraic line bundle over $X_{\mathbb{R}} \times \operatorname{Pic}^{d}\left(X_{\mathbb{R}}\right)$ if and only if the Euler characteristic $\chi(L)$ is odd for $L \in \operatorname{Pic}^{d}\left(X_{\mathbb{R}}\right)$. There is a universal quaternionic algebraic line bundle over $X \times \operatorname{Pic}^{d}(X)$ if and only if the degree $d$ is odd. (Quaternionic algebraic vector bundles are defined only on a complexification.)

Take integers $r$ and $d$ such that $r \geq 2$, and $d$ is coprime to $r$. Let $\mathcal{M}_{X_{\mathbb{R}}}(r, d)$ (respectively, $\mathcal{M}_{X}(r, d)$ ) be the moduli space of stable vector bundles over $X_{\mathbb{R}}$ (respectively, $X$ ) of rank $r$ and degree $d$. We prove that there is a universal real algebraic vector bundle over $X_{\mathbb{R}} \times \mathcal{M}_{X_{\mathbb{R}}}(r, d)$ if and only if $\chi(E)$ is odd for $E \in \mathcal{M}_{X_{\mathbb{R}}}(r, d)$. There is a universal quaternionic vector bundle over $X \times \mathcal{M}_{X}(r, d)$ if and only if the degree $d$ is odd.

The cases where $X_{\mathbb{R}}$ is geometrically reducible or $X_{\mathbb{R}}$ has real points are also investigated.
\end{abstract}

\section{INTRODUCTION}

Let $Y$ be a compact connected Riemann surface of genus $g$. For any integers $r \geq 2$ and $d$, let $\mathcal{M}_{Y}(r, d)$ be the moduli space of stable vector bundles over $Y$ of rank $r$ and degree $d$. A Poincaré bundle (also called a universal bundle) is an algebraic vector bundle $\mathcal{E} \longrightarrow Y \times \mathcal{M}_{Y}(r, d)$ with the property that for each point $z \in \mathcal{M}_{Y}(r, d)$, the vector bundle $\left.\mathcal{E}\right|_{Y \times\{z\}}$ on $Y$ is in the isomorphism class of vector bundles defined by $z$. Similarly, a Poincaré line bundle $\mathcal{L} \longrightarrow Y \times \operatorname{Pic}^{d}(Y)$ is an algebraic line bundle with the property that for each point $z \in \operatorname{Pic}^{d}(Y)$, the restriction $\left.\mathcal{L}\right|_{Y \times\{z\}}$ is in the isomorphism class of line bundles defined by $z$. Poincaré vector bundles are very useful objects; for example, they are used in computing the cohomology of $\mathcal{M}_{Y}(r, d)([\mathrm{MN}],[\mathrm{JK}])$.

There is a Poincaré line bundle on $Y \times \operatorname{Pic}^{d}(Y)$ for each $d$. If $g \geq 2$, it is known that there is a universal vector bundle over $Y \times \mathcal{M}_{Y}(r, d)$ if and only if $d$ is coprime to $r$. A similar statement holds for the moduli space of stable vector bundles over $Y$ with fixed determinant. Our aim here is to address similar questions for curves defined over the real numbers.

Let $X_{\mathbb{R}}$ be an irreducible smooth projective curve defined over $\mathbb{R}$. If $X_{\mathbb{R}}$ is geometrically reducible, or if $X_{\mathbb{R}}$ has a real point, then it is straightforward to

Received by the editors September 10, 2009 and, in revised form, January 22, 2010 and January $25,2010$.

2010 Mathematics Subject Classification. Primary 14F05, 14 P99.

Key words and phrases. Real curve, moduli space, real universal bundle.

(C)2011 American Mathematical Society 
answer the question of existence of a universal bundle (see Section 2.3 for the details). So assume that

(1) $X_{\mathbb{R}}$ is geometrically irreducible, and

(2) $X_{\mathbb{R}}$ does not have any real points.

Let $X:=X_{\mathbb{R}} \times_{\mathbb{R}} \mathbb{C}$ be the complex curve obtained by base change. The conjugation of $\mathbb{C}$ gives an anti-holomorphic involution $\sigma$ of $X$. Real algebraic vector bundles over $X_{\mathbb{R}}$ are the same as complex algebraic vector bundles $E \longrightarrow X$ together with an algebraic isomorphism $\sigma_{E}: E \longrightarrow \sigma^{*} \bar{E}$ with $\sigma_{E} \circ \sigma^{*}\left(\bar{\sigma}_{E}\right)=\operatorname{Id}_{E}$. Using the canonical $C^{\infty}$ isomorphism of $E$ with $\bar{E}$, an isomorphism of $E$ with $\sigma^{*} \bar{E}$ is given by an anti-holomorphic automorphism of the total space of $E$ lifting $\sigma$ which is conjugate linear on the fibers. The above condition $\sigma_{E} \circ \sigma^{*}\left(\bar{\sigma}_{E}\right)=\operatorname{Id}_{E}$ is equivalent to the condition that this automorphism is an involution.

A quaternionic vector bundle over $X$ is defined to be an algebraic vector bundle $E \longrightarrow X$ equipped with an algebraic isomorphism $\sigma_{E}: E \longrightarrow \sigma^{*} \bar{E}$ such that $\sigma_{E} \circ \sigma^{*}\left(\bar{\sigma}_{E}\right)=-\operatorname{Id}_{E}$. Quaternionic vector bundles are defined only on the complexification $X$ and not on $X_{\mathbb{R}}$. Since the automorphism $-\operatorname{Id}_{E}$ acts trivially on the projective bundle $\mathbb{P}(E)$ associated to $E$, a quaternionic vector bundle on $X$ defines a real projective bundle over $X_{\mathbb{R}}$.

These descriptions of real and quaternionic vector bundles hold for any variety defined over $\mathbb{R}$. Real algebraic universal vector bundles for $X_{\mathbb{R}}$ will be identified with the universal vector bundles for $X$ equipped with a lift of the anti-holomorphic involution of the base; they will be called real universal vector bundles.

For a vector bundle $E$ on $X$ of rank $r$ and degree $d$, define

$$
\chi(E):=\operatorname{dim} H^{0}(X, E)-\operatorname{dim} H^{1}(X, E) .
$$

By Riemann-Roch, this is expressed as

$$
d-r(\operatorname{genus}(X)-1) \text {. }
$$

It turns out that, contrary to the complex case, there are obstructions for the existence of a real or quaternionic universal line bundle over $X \times \operatorname{Pic}^{d}(X)$. More precisely, we prove the following:

Theorem 1.1. There is a real universal line bundle over $X \times \operatorname{Pic}^{d}(X)$ if and only if $\chi(L)$ is odd for $L \in \operatorname{Pic}^{d}(X)$.

There is a quaternionic universal line bundle over $X \times \operatorname{Pic}^{d}(X)$ if and only if $d$ is odd.

See Propositions 3.3, 3.4 and 3.5 for a proof of Theorem 1.1 ,

Take any integer $r \geq 2$. Let $d$ be any integer coprime to $r$ (as mentioned above, there is no universal vector bundle, even over the complex numbers, if g.c.d. $(r, d) \neq$ $1)$. Let $\mathcal{M}_{X}(r, d)$ be the moduli space of stable vector bundles over $X$ of rank $r$ and degree $d$.

Theorem 1.2. There is a real universal vector bundle over $X \times \mathcal{M}_{X}(r, d)$ if and only if $\chi(E)$ is odd for $E \in \mathcal{M}_{X}(r, d)$.

There is a quaternionic universal vector bundle over $X \times \mathcal{M}_{X}(r, d)$ if and only if the degree $d$ is odd.

See Propositions 4.2, 4.3, 4.4, and 4.5 for a proof of Theorem 1.2 .

A similar result holds for moduli spaces of vector bundles with fixed determinant; see Section 5 . 
We note that, somewhat ironically, one can have a universal real (or quaternionic) bundle on $X \times \operatorname{Pic}^{d}(X)$ or $X \times \mathcal{M}_{X}(r, d)$ without there being a corresponding real (or quaternionic) bundle over $X$. Indeed, the constraint for the existence of a real bundle on $X$ is that the degree $d$ be even; for a quaternionic bundle, $\chi(E)$ must be even (see $[\mathrm{BHH}]$ ); these can be contrasted with the conditions in the theorems above.

Instead of the involution $E \longrightarrow \sigma^{*} \bar{E}$, one may also consider the involution of the moduli space defined by $E \longrightarrow \sigma^{*} \bar{E}^{*}$. Note that degree $(E)=0$ if $E$ and $\sigma^{*} \bar{E}^{*}$ lie in the same moduli space. Unitary flat vector bundles on nonorientable surfaces are extensively studied; see [HJ], $\mathrm{HL}$. The holomorphic vector bundles corresponding to these flat bundles are fixed points of the involution $E \longrightarrow \sigma^{*} \bar{E}^{*}$. Since there is no universal vector bundle over $X \times \mathcal{M}_{X}(r, 0)$ if $r \geq 2$, there is no real or quaternionic universal vector bundle over $X \times \mathcal{M}_{X}(r, 0)$ for this involution.

\section{ReAl CURVES AND UNIVERSAL BUNDLES}

2.1. Universal bundles. Let $Y$ be a compact connected Riemann surface. Let $J(Y)$ be the Jacobian of $Y$, which is an abelian variety that parametrizes the isomorphism classes of topologically trivial holomorphic line bundles over $Y$. For any integer $d$, let $\operatorname{Pic}^{d}(Y)$ denote the torsor for $J(Y)$ that parametrizes the isomorphism classes of holomorphic line bundles over $Y$ of degree $d$. A Poincaré line bundle over $Y \times \operatorname{Pic}^{d}(Y)$ is a holomorphic line bundle

$$
\mathcal{L} \longrightarrow Y \times \operatorname{Pic}^{d}(Y)
$$

such that for each point $z \in \operatorname{Pic}^{d}(Y)$ the line bundle $\left.\mathcal{L}\right|_{Y \times\{z\}} \longrightarrow Y$ is in the isomorphism class parametrized by $z$. There is a Poincaré line bundle over $Y \times$ $\operatorname{Pic}^{d}(Y)$. If we fix a point $y_{0} \in Y$, then there is a unique, up to an isomorphism, Poincaré line bundle over $Y \times \operatorname{Pic}^{d}(Y)$ which is trivial over $\left\{y_{0}\right\} \times \operatorname{Pic}^{d}(Y)$; see [ACGH, Ch. IV , §2] for the details. A Poincaré line bundle is also called a universal line bundle.

Let $g$ be the genus of $Y$.

Fix an integer $r \geq 2$ and let $d$ be any integer. Let $\mathcal{M}_{Y}(r, d)$ denote the moduli space of stable vector bundles over $Y$ of rank $r$ and degree $d$. This moduli space, which was first constructed by Mumford, $[\mathrm{Mu}$, is an irreducible smooth quasiprojective variety over $\mathbb{C}$. If $g=0$, then $\mathcal{M}_{Y}(r, d)$ is an empty set; this follows immediately from a theorem of Grothendieck which says that any holomorphic vector bundle over $\mathbb{C P}^{1}$ splits into a direct sum of holomorphic line bundles $\mathrm{Gr}$. If $g=1$, and g.c.d. $(r, d) \neq 1$, then $\mathcal{M}_{Y}(r, d)$ is an empty set; if g.c.d. $(r, d)=1$, then, up to tensoring with a line bundle, there is exactly one stable vector bundle over $Y$ of rank $r$ and degree $d$ (see [At1]). If $g \geq 2$, then the dimension of $\mathcal{M}_{Y}(r, d)$ is $r^{2}(g-1)+1$.

When we consider the moduli space $\mathcal{M}_{Y}(r, d)$, it will always be assumed that $g \geq 2$.

An algebraic vector bundle

$$
\mathcal{E} \longrightarrow Y \times \mathcal{M}_{Y}(r, d)
$$

is called universal if for each point $z \in \mathcal{M}_{Y}(r, d)$, the vector bundle $\left.\mathcal{E}\right|_{Y \times\{z\}} \longrightarrow Y$ is in the isomorphism class parametrized by $z$. 
There is a universal vector bundle over $Y \times \mathcal{M}_{Y}(r, d)$ if and only if g.c.d. $(r, d)=$ 1 Ra (see also $\mathrm{Ne}$ ). In Section 2.3 we recall a construction of the universal vector bundle under the assumption that the degree is coprime to the rank.

2.2. Real curves: real and quaternionic bundles. Let $X_{\mathbb{R}}$ be an irreducible smooth projective curve defined over the field of real numbers. The corresponding complex curve $X:=X_{\mathbb{R}} \times_{\mathbb{R}} \mathbb{C}$ is a compact Riemann surface. The automorphism of $\mathbb{C}$ defined by $z \longmapsto \bar{z}$ produces an anti-holomorphic involution

$$
\sigma: X \longrightarrow X \text {. }
$$

If $X_{\mathbb{R}}$ is geometrically irreducible, then $X$ is connected. If $X_{\mathbb{R}}$ is not geometrically irreducible, then $X$ is a disjoint union

$$
X=S \cup \bar{S},
$$

where $S$ is a compact connected Riemann surface, and $\bar{S}$ is the conjugate of $S$, meaning that the smooth manifold underlying $\bar{S}$ coincides with that of $S$, while the almost complex structure of $\bar{S}$ is $-J_{S}$, where $J_{S}$ is the almost complex structure of $S$. So the identity map $S \longrightarrow \bar{S}$ is anti-holomorphic, and this map coincides with $\sigma$.

Let $Z$ be a variety defined over $\mathbb{R}$; let $Z_{\mathbb{C}}:=Z \times_{\mathbb{R}} \mathbb{C}$ be the corresponding complex variety. Let

$$
\sigma_{Z}: Z_{\mathbb{C}} \longrightarrow Z_{\mathbb{C}}
$$

be the anti-holomorphic involution given by the automorphism of $\mathbb{C}$ defined by $z \longmapsto \bar{z}$. Giving a real algebraic vector bundle over $Z$ is equivalent to giving a complex algebraic vector bundle $E \longrightarrow Z_{\mathbb{C}}$ together with an algebraic isomorphism of vector bundles

$$
\eta: E \longrightarrow \sigma_{Z}^{*} \bar{E}
$$

such that the composition

$$
E \stackrel{\eta}{\longrightarrow} \sigma_{Z}^{*} \bar{E} \stackrel{\sigma_{Z}^{*} \bar{\eta}}{\longrightarrow} \sigma_{Z}^{*} \overline{\sigma_{Z}^{*} \bar{E}}=E
$$

is the identity map of $E$. Note that this condition means that there is an involution lifting $\sigma_{Z}$.

The above correspondence between real algebraic vector bundles on $Z$ and complex algebraic vector bundles on the complexification $Z_{\mathbb{C}}$ equipped with an involution lifting $\sigma_{Z}$ will be used throughout without further clarification.

For convenience, sometimes a real algebraic vector bundle over $Z$ will also be called a real algebraic vector bundle on $Z_{\mathbb{C}}$ (this happens when we refer to both real and quaternionic vector bundles in the same sentence).

In the same vein, a quaternionic vector bundle on $Z_{\mathbb{C}}$ is a complex algebraic vector bundle

$$
E \longrightarrow Z_{\mathbb{C}}
$$

together with an algebraic isomorphism

$$
\eta: E \longrightarrow \sigma_{Z}^{*} \bar{E}
$$

such that the composition

$$
E \stackrel{\eta}{\longrightarrow} \sigma_{Z}^{*} \bar{E} \stackrel{\sigma_{Z}^{*} \bar{\eta}}{\longrightarrow} \sigma_{Z}^{*} \overline{\sigma_{Z}^{*} \bar{E}}=E
$$

is multiplication by -1 . 
Note that the quaternionic vector bundles are defined only on the complexification.

For any integer $d$, let $\operatorname{Pic}^{d}\left(X_{\mathbb{R}}\right)_{\mathbb{C}}:=\operatorname{Pic}^{d}\left(X_{\mathbb{R}}\right) \times_{\mathbb{R}} \mathbb{C}$ be the complexification of the Picard variety of $X_{\mathbb{R}}$. So, if $X_{\mathbb{R}}$ is geometrically irreducible, then

$$
\operatorname{Pic}^{d}\left(X_{\mathbb{R}}\right)_{\mathbb{C}}=\operatorname{Pic}^{d}(X) .
$$

If $X_{\mathbb{R}}$ is not geometrically irreducible, and if $d$ is odd, then $\operatorname{Pic}^{d}\left(X_{\mathbb{R}}\right)_{\mathbb{C}}$ is the empty set. If $X_{\mathbb{R}}$ is not geometrically irreducible, and if $d$ is even, then

$$
\operatorname{Pic}^{d}\left(X_{\mathbb{R}}\right)_{\mathbb{C}}=\operatorname{Pic}^{d / 2}(S) \times \operatorname{Pic}^{d / 2}(\bar{S}),
$$

where $S$ and $\bar{S}$ are as in (2.2).

Let

$$
\tilde{\sigma}: \operatorname{Pic}^{d}\left(X_{\mathbb{R}}\right)_{\mathbb{C}} \longrightarrow \operatorname{Pic}^{d}\left(X_{\mathbb{R}}\right)_{\mathbb{C}}
$$

be the anti-holomorphic diffeomorphism that sends any holomorphic line bundle $L$ on $X$ to $\sigma^{*} \bar{L}$, where $\sigma$ is the anti-holomorphic involution in (2.1). This $\widetilde{\sigma}$ is clearly an involution, and it coincides with the involution given by the automorphism $z \longmapsto \bar{z}$ of $\mathbb{C}$.

Note that both quaternionic and real line bundles over $X$ of degree $d$ are represented by real points of $\operatorname{Pic}^{d}\left(X_{\mathbb{R}}\right)_{\mathbb{C}}$.

Now we consider stable vector bundles of higher ranks.

If $X_{\mathbb{R}}$ is geometrically irreducible, an algebraic vector bundle $E$ over $X_{\mathbb{R}}$ will be called stable if for every proper subbundle $F \subset E$ of positive rank, the following inequality holds:

$$
\text { degree }(F) / \operatorname{rank}(F)<\operatorname{degree}(E) / \operatorname{rank}(E) \text {. }
$$

So if $E$ is stable, then the corresponding vector bundle $E \bigotimes_{\mathbb{R}} \mathbb{C}$ over $X$ is polystable. For a real algebraic vector bundle $E \longrightarrow X_{\mathbb{R}}$, from the uniqueness of the HarderNarasimhan filtration it follows that each term in the Harder-Narasimhan filtration of $E \bigotimes_{\mathbb{R}} \mathbb{C}$ over $X_{\mathbb{R}} \times_{\mathbb{R}} \mathbb{C}$ is real, meaning it is preserved by the involution of $E \bigotimes_{\mathbb{R}} \mathbb{C}$. We note that if $d$ is coprime to $r$, then $E$ is stable if and only if $E \bigotimes_{\mathbb{R}} \mathbb{C}$ on $X$ is stable.

If $X_{\mathbb{R}}$ is not geometrically irreducible, an algebraic vector bundle $E$ over $X_{\mathbb{R}}$ will be called stable if the restriction of $E \bigotimes_{\mathbb{R}} \mathbb{C}$ to the component $S$ in (2.2) is stable. Note that the restriction of $E \bigotimes_{\mathbb{R}} \mathbb{C}$ to $S$ is stable if and only if the restriction of $E \otimes_{\mathbb{R}} \mathbb{C}$ to $\bar{S}$ is stable.

Let $g:=H^{1}\left(X_{\mathbb{R}}, \mathcal{O}_{X_{\mathbb{R}}}\right)$ be the genus of $X_{\mathbb{R}}$. As mentioned before, while considering higher rank bundles it is assumed that $g \geq 2$. We further assume that $g \geq 4$ if $X_{\mathbb{R}}$ is not geometrically irreducible. This condition is equivalent to the condition that the genus of $S$ in (2.2) is at least two.

Let $\mathcal{M}_{X_{\mathbb{R}}}(r, d)$ be the moduli space of stable vector bundles over $X_{\mathbb{R}}$ of rank $r$ and degree $d$. Let

$$
\widetilde{\mathcal{M}}_{X}(r, d)=\mathcal{M}_{X_{\mathbb{R}}}(r, d) \times_{\mathbb{R}} \mathbb{C}
$$

be the complexification. Assume that $X_{\mathbb{R}}$ is geometrically irreducible. Let $\mathcal{M}_{X}(r, d)$ be the moduli space of stable vector bundles over $X$ of rank $r$ and degree $d$. From the construction of the moduli space it follows that $\mathcal{M}_{X}(r, d)$ is a Zariski open subset of $\widetilde{\mathcal{M}}_{X}(r, d)$. But if $d$ is coprime to $r$, then $\mathcal{M}_{X}(r, d)$ coincides with $\widetilde{\mathcal{M}}_{X}(r, d)$. 
If $X_{\mathbb{R}}$ is not geometrically irreducible, and if $d$ is odd, then $\widetilde{\mathcal{M}}_{X}(r, d)$ is the empty set. If $X_{\mathbb{R}}$ is not geometrically irreducible, and if $d$ is even, then

$$
\widetilde{\mathcal{M}}_{X}(r, d)=\mathcal{M}_{S}(r, d / 2) \times \mathcal{M}_{\bar{S}}(r, d / 2),
$$

where $S$ and $\bar{S}$ are as in (2.2); recall that genus $(S) \geq 2$. Let

$$
\widetilde{\sigma}: \widetilde{\mathcal{M}}_{X}(r, d) \longrightarrow \widetilde{\mathcal{M}}_{X}(r, d)
$$

be the anti-holomorphic diffeomorphism defined by $E \longmapsto \sigma^{*} \bar{E}$, where $\sigma$ is the involution in (2.1). We note that $\widetilde{\sigma}$ coincides with the involution of $\widetilde{\mathcal{M}}_{X}(r, d)$ given by the automorphism $z \longmapsto \bar{z}$ of $\mathbb{C}$.

Note that both quaternionic and real stable vector bundles over $X$ of rank $r$ and degree $d$ are represented by real points of $\widetilde{\mathcal{M}}_{X}(r, d)$.

2.3. Real and quaternionic universal bundles. First assume that $X_{\mathbb{R}}$ is geometrically irreducible. A real universal line bundle over $X \times \operatorname{Pic}^{d}(X)$ is a universal line bundle

$$
\mathcal{L} \longrightarrow X \times \operatorname{Pic}^{d}(X)
$$

equipped with a holomorphic

$$
\sigma_{P}: \mathcal{L} \longrightarrow(\sigma \times \widetilde{\sigma})^{*} \overline{\mathcal{L}},
$$

where $\sigma$ and $\widetilde{\sigma}$ are the involutions in (2.1) and (2.4), respectively, such that $\sigma_{P} \circ$ $\sigma_{P}=\operatorname{Id}_{\mathcal{L}}$. The definition of a quaternionic universal bundle is the same, except that $\sigma_{P} \circ \sigma_{P}=-\operatorname{Id}_{\mathcal{L}}$.

Now assume that $X_{\mathbb{R}}$ is not geometrically irreducible. Take $d$ to be even, say $d=2 d_{0}$ (recall that $\operatorname{Pic}^{d}\left(X_{\mathbb{R}}\right)_{\mathbb{C}}$ is the empty set when $d$ is odd).

A real universal line bundle over $X \times \operatorname{Pic}^{d}\left(X_{\mathbb{R}}\right)_{\mathbb{C}}$ is a universal line bundle

$$
\mathcal{L} \longrightarrow S \times \operatorname{Pic}^{d_{0}}(S)
$$

where $S$ is the Riemann surface in (2.2). Note that the universal line bundle over $\bar{S} \times \operatorname{Pic}^{d_{0}}(\bar{S})$ is uniquely determined by $\mathcal{L} ;$ more precisely, it is the pullback $(\sigma \times \widetilde{\sigma})^{*} \overline{\mathcal{L}}$.

Since a universal line bundle over $S \times \operatorname{Pic}^{d_{0}}(S)$ exists, we conclude that there is a real (or quaternionic; we just have to change the sign of the isomorphism over the component $S \times \operatorname{Pic}^{d_{0}}(S)$ of $\left.\left(S \times \operatorname{Pic}^{d_{0}}(S)\right) \cup\left(\bar{S} \times \operatorname{Pic}^{d_{0}}(\bar{S})\right)\right)$ universal line bundle over $X \times \operatorname{Pic}^{d}\left(X_{\mathbb{R}}\right)_{\mathbb{C}}$ if $X_{\mathbb{R}}$ is not geometrically irreducible.

The real points of $X_{\mathbb{R}}$ are the fixed points of the involution $\sigma$ of $X$. Assume that $X_{\mathbb{R}}$ has a real point $x_{0}$ (note that this implies that $X_{\mathbb{R}}$ is geometrically irreducible). As mentioned in Section 2.1, there is a unique, up to an isomorphism, universal line bundle over $X \times \operatorname{Pic}^{d}(X)$ which is trivial on $\left\{x_{0}\right\} \times \operatorname{Pic}^{d}(X)$. Let $\mathcal{L}_{d} \longrightarrow X \times$ $\operatorname{Pic}^{d}(X)$ be this universal line bundle. Fix a trivialization of $\mathcal{L}_{d}$ over $\left\{x_{0}\right\} \times \operatorname{Pic}^{d}(X)$. From the uniqueness of $\mathcal{L}_{d}$ it follows that there is a unique involution

$$
\mathcal{L}_{d} \longrightarrow(\sigma \times \widetilde{\sigma})^{*} \overline{\mathcal{L}_{d}}
$$

whose restriction to $\left\{x_{0}\right\} \times \operatorname{Pic}^{d}(X)$ is the conjugation of $\mathbb{C}$ with respect to the chosen trivialization; the involution $\widetilde{\sigma}$ is constructed in (2.4). In other words, $\mathcal{L}_{d}$ gets a real structure. Consequently, if $\sigma$ has a fixed point, there is a real universal line bundle over $X \times \operatorname{Pic}^{d}(X)$.

Now we consider the moduli spaces of stable vector bundles of higher rank.

First assume that $X_{\mathbb{R}}$ is not geometrically irreducible. Take the integer $d$ to be even, say $d=2 d_{0}$ (recall that $\widetilde{\mathcal{M}}_{X}(r, d)$ in (2.5) is the empty set when $d$ is odd). 
A real universal vector bundle over $X \times \widetilde{\mathcal{M}}_{X}(r, d)$ is a universal vector bundle

$$
\mathcal{E} \longrightarrow S \times \mathcal{M}_{S}\left(r, d_{0}\right)
$$

where $S$ is the Riemann surface in (2.2); as before, $\mathcal{E}$ gives the universal vector bundle $(\sigma \times \widetilde{\sigma})^{*} \overline{\mathcal{E}}$ over $\bar{S} \times \mathcal{M}_{\bar{S}}\left(r, d_{0}\right)$.

As mentioned in Section 2.1, there is a universal vector bundle over $S \times \mathcal{M}_{S}\left(r, d_{0}\right)$ if and only if $d_{0}$ is coprime to $r$. Hence, if $X_{\mathbb{R}}$ is not geometrically irreducible, there is a real (or quaternionic) universal vector bundle over $X \times \widetilde{\mathcal{M}}_{X}\left(r, 2 d_{0}\right)$ if and only if $d_{0}$ is coprime to $r$.

Now assume that $X_{\mathbb{R}}$ is geometrically irreducible. A real universal vector bundle over $X \times \mathcal{M}_{X}(r, d)$ is a universal vector bundle

$$
\mathcal{E} \longrightarrow X \times \mathcal{M}_{X}(r, d)
$$

equipped with a conjugate-linear involution

$$
\mathcal{E} \longrightarrow \mathcal{E}
$$

lifting $\sigma \times \widetilde{\sigma}$, where $\sigma$ and $\widetilde{\sigma}$ are the involutions constructed in (2.1) and (2.6), respectively. The square of this lift must be the identity; for a universal quaternionic vector bundle, one has the same type of lift, but now one asks that the square be minus the identity.

We will show that if $\sigma$ has a fixed point, and $d$ is coprime to $r$, then there is a real universal vector bundle over $X \times \mathcal{M}_{X}(r, d)$. For that we need to recall a construction of the universal vector bundle over the complex numbers under the assumption that $d$ is coprime to $r$.

As before, assume that $X_{\mathbb{R}}$ is geometrically irreducible. Assume that $d$ is coprime to $r$. Set

$$
\chi:=d+r(1-g),
$$

where $g$ is the genus of $X$. Note that $\chi$ is the Euler characteristic of any vector bundle on $X$ lying in $\mathcal{M}_{X}(r, d)$. Since $d$ is coprime to $r$, there are integers $a$ and $b$ such that

$$
a r+b \chi=-1
$$

The moduli space $\mathcal{M}_{X}(r, d)$ is a quotient of a Quot scheme $\left.\mathcal{Q}(\underline{\mathrm{Mu}}]\right)$. Let

$$
\mathcal{E} \longrightarrow X \times \mathcal{Q}
$$

be the tautological universal vector bundle. Fix a point $x_{0}$ in $X$. Let

$$
\mathcal{E}_{x_{0}} \longrightarrow\left\{x_{0}\right\} \times \mathcal{Q}=\mathcal{Q}
$$

be the restriction. Let

$$
\mathcal{D}:=\left(\operatorname{det} R^{0} p_{*} \mathcal{E}\right) \otimes\left(\operatorname{det} R^{1} p_{*} \mathcal{E}\right)^{*} \longrightarrow \mathcal{Q}
$$

be the determinant line bundle, where $p: X \times \mathcal{Q} \longrightarrow \mathcal{Q}$ is the natural projection. Then the vector bundle

$$
\mathcal{E} \otimes\left(\bigwedge^{r} p^{*} \mathcal{E}_{x_{0}}\right)^{\otimes a} \otimes p^{*} \mathcal{D}^{\otimes b} \longrightarrow X \times \mathcal{Q}
$$

descends to a universal vector bundle over $X \times \mathcal{M}_{X}(r, d)$, where $a$ and $b$ are as in (2.9). From (2.8) it follows that the multiplication action of the nonzero scalars on the vector bundle in (2.9) is trivial. We recall that the vector bundle in (2.9) descends to $X \times \mathcal{M}_{X}(r, d)$ because $\mathbb{C}^{*}$ acts trivially on it. 
The Quot scheme $\mathcal{Q}$ can be so chosen that it has a real structure; indeed, one is looking at quotients of a fixed real bundle, which can be chosen to be real. Then the vector bundle $\mathcal{E} \longrightarrow X \times \mathcal{Q}$ has a real structure. If the point $x_{0}$ is fixed by $\sigma$, the universal vector bundle over $X \times \mathcal{M}_{X}(r, d)$ constructed above has a real structure.

If $a$ in (2.9) is an even integer, then the vector bundle in (2.9) can be replaced by

$$
\left.\mathcal{E} \otimes\left(\bigwedge^{r} p^{*} \mathcal{E}_{x_{0}}\right)^{\otimes a / 2} \otimes\left(\bigwedge^{r} p^{*} \mathcal{E}_{\sigma\left(x_{0}\right.}\right)\right)^{\otimes a / 2} \otimes \mathcal{D}^{\otimes b} \longrightarrow X \times \mathcal{Q},
$$

which also descends to $X \times \mathcal{M}_{X}(r, d)$ as a universal vector bundle for the same reason. If the Quot scheme has a real structure, this descended vector bundle also has a real structure.

So we have the following lemma.

Lemma 2.1. Assume that $X_{\mathbb{R}}$ is geometrically irreducible. Also, assume that $d$ is coprime to $r$.

If $\sigma$ has a fixed point, then there is a real universal vector bundle over $X \times$ $\mathcal{M}_{X}(r, d)$.

If the integer a in (2.8) is even, then there is a real universal vector bundle over $X \times \mathcal{M}_{X}(r, d)$.

One could try the same approach for quaternionic structures: the universal bundle over the Quot scheme can be made quaternionic, but unfortunately the technique for having it descend to the moduli space does not go through.

Henceforth, we will always make the following two assumptions:

(1) $X_{\mathbb{R}}$ is geometrically irreducible, and

(2) $X_{\mathbb{R}}$ does not have any real points.

We have seen above that the question of the existence of a real universal bundle is settled if any of the above two conditions fails.

\section{LINE BUNDLES}

3.1. Existence of universal bundles. The following lemma gives an explicit universal line bundle.

Lemma 3.1. There is a natural real universal line bundle over $X \times \operatorname{Pic}^{g-2}(X)$.

Proof. On $\mathrm{Pic}^{g-1}(X)$, there is a canonical theta hypersurface $\Theta$ that parametrizes all line bundles $\zeta \longrightarrow X$ of degree $g-1$ such that $H^{0}(X, \zeta) \neq 0$. If

$$
\mathcal{L}_{g-1} \longrightarrow X \times \operatorname{Pic}^{g-1}(X)
$$

is a universal line bundle, then the line bundle $\mathcal{O}_{\mathrm{Pic}^{g-1}(X)}(-\Theta)$ is the determinant line bundle

$$
\operatorname{det} \mathcal{L}_{g-1}:=\left(\operatorname{det} p_{2 *} \mathcal{L}_{g-1}\right) \otimes\left(\operatorname{det} R^{1} p_{2 *} \mathcal{L}_{g-1}\right)^{*} \longrightarrow \operatorname{Pic}^{g-1}(X),
$$

where $p_{2}$ is the projection of $X \times \operatorname{Pic}^{g-1}(X)$ to $\operatorname{Pic}^{g-1}(X)$. Since $\chi(L)=0$ for any $L \in \operatorname{Pic}^{g-1}(X)$, the determinant line bundle $\operatorname{det} \mathcal{L}_{g-1}$ is independent of the choice of $\mathcal{L}_{g-1}$.

Let

$$
\phi: X \times \operatorname{Pic}^{g-2}(X) \longrightarrow \operatorname{Pic}^{g-1}(X)
$$


be the map defined by $(x, L) \longmapsto L \otimes \mathcal{O}_{X}(x)$. Define a line bundle

$$
\mathcal{L}:=\phi^{*} \mathcal{O}_{\operatorname{Pic}^{g-1}(X)}(-\Theta) \otimes p_{X}^{*} K_{X} \longrightarrow X \times \operatorname{Pic}^{g-2}(X),
$$

where $K_{X}$ is the holomorphic cotangent bundle of $X$, and $p_{X}$ is the projection of $X \times \operatorname{Pic}^{g-2}(X)$ to $X$. We will show that $\mathcal{L}$ is a universal line bundle.

For that, take any holomorphic line bundle

$$
\zeta \longrightarrow X
$$

of degree $g-2$. Consider the line bundle

$$
\widetilde{\zeta}:=\left(q_{1}^{*} \zeta\right) \otimes \mathcal{O}_{X \times X}(\Delta) \longrightarrow X \times X,
$$

where $q_{i}, i=1,2$, is the projection of $X \times X$ to the $i$-th factor, and $\Delta \subset X \times X$ is the diagonal divisor. From the above-mentioned identification of $\mathcal{O}_{\mathrm{Pic}^{g-1}(X)}(-\Theta)$ as a determinant line bundle, it follows that

$$
\left.\phi^{*} \mathcal{O}_{\operatorname{Pic}^{g-1}(X)}(-\Theta)\right|_{X \times\{\zeta\}}=\operatorname{det} \widetilde{\zeta}:=\left(\operatorname{det} R^{0} q_{2 *} \widetilde{\zeta}\right) \otimes\left(\operatorname{det} R^{1} q_{2 *} \widetilde{\zeta}\right)^{*},
$$

where $\phi$ is the map in (3.1).

In view of (3.3), to prove that $\mathcal{L}$ is a universal line bundle it suffices to show that

$$
\zeta=(\operatorname{det} \widetilde{\zeta}) \otimes K_{X}
$$

To prove (3.4), we use [BM, p. 368, Lemma 6$]$ for the family of line bundles $\widetilde{\zeta}$ over $X$ parametrized by the second factor of $X \times X$. Given a pair of algebraic line bundles $\eta_{1}$ and $\eta_{2}$ on an algebraic family of curves, the Deligne pairing $\left\langle\eta_{1}, \eta_{2}\right\rangle$ is a line bundle on the parameter space (see $[\mathrm{De}, \mathrm{BM}]$ for its construction). The line bundle $\left\langle q_{1}^{*} \zeta, \mathcal{O}_{X \times X}(\Delta)\right\rangle$ is isomorphic to $\zeta$ (see [BM p. 367, Proposition 5(c)]). Now [BM] p. 368, Lemma 6] says that

$$
\zeta=(\operatorname{det} \widetilde{\zeta}) \otimes\left(\operatorname{det} \mathcal{O}_{X \times X}(\Delta)\right)^{*}
$$

(all other line bundles in $\overline{\mathrm{BM}}$, p. 368, Lemma 6] are trivial in our case).

Consider the short exact sequence of sheaves on $X \times X$ :

$$
\left.0 \longrightarrow \mathcal{O}_{X \times X} \longrightarrow \mathcal{O}_{X \times X}(\Delta) \longrightarrow \mathcal{O}_{X \times X}(\Delta)\right|_{\Delta} \longrightarrow 0 .
$$

The Poincaré adjunction formula says that the restriction of the line bundle $\mathcal{O}_{X \times X}(\Delta)$ to $\Delta$ is the tangent bundle $T X$. Hence from (3.6),

$$
\operatorname{det} \mathcal{O}_{X \times X}(\Delta)=T X,
$$

where $T X$ is the holomorphic tangent bundle. Now (3.5) implies (3.4). Therefore, $\mathcal{L}$ is a universal line bundle over $X \times \operatorname{Pic}^{g-2}(X)$.

From the construction of $\mathcal{L}$ it follows immediately that there is a natural involution of $\mathcal{L}$ lifting the anti-holomorphic involution $\sigma \times \widetilde{\sigma}$ of $X \times \operatorname{Pic}^{g-2}(X)$. Therefore, $\mathcal{L}$ is a real universal line bundle. This completes the proof of the lemma.

We recall that a real (respectively, quaternionic) algebraic line bundle on $X_{\mathbb{R}}$ is a holomorphic line bundle on $X$ equipped with an involution lifting $\sigma$, whose square is the identity (respectively, minus the identity).

Lemma 3.2. Let $d$ be an integer such that there is a real universal line bundle on $X \times \operatorname{Pic}^{d}(X)$. Let $L$ be a real (respectively, quaternionic) line bundle on $X$ of degree $d_{1}$. Then there is a real (respectively, quaternionic) universal line bundle on $X \times \operatorname{Pic}^{d+d_{1}}(X)$. 
Similarly, let $d$ be an integer such that there is a quaternionic universal line bundle on $X \times \operatorname{Pic}^{d}(X)$. Let $L$ be a real (respectively, quaternionic) line bundle on $X$ of degree $d_{1}$. Then there is a quaternionic (respectively, real) universal line bundle on $X \times \operatorname{Pic}^{d+d_{1}}(X)$.

Proof. Let $\mathcal{L} \longrightarrow X \times \operatorname{Pic}^{d}(X)$ be a real universal line bundle. Let

$$
\phi: \operatorname{Pic}^{d+d_{1}}(X) \longrightarrow \operatorname{Pic}^{d}(X)
$$

be the morphism defined by $L^{\prime} \longmapsto L^{\prime} \otimes L^{*}$. Then $\left(\operatorname{Id}_{X} \times \phi\right)^{*} \mathcal{L} \otimes p_{X}^{*} L$, where $p_{X}$ is the projection of $X \times \operatorname{Pic}^{d+d_{1}}(X)$ to $X$, is naturally a real (respectively, quaternionic) universal line bundle if $L$ is real (respectively, quaternionic). The same procedure works for the second half of the proposition.

For any point $x_{0} \in X$, the line bundle $\mathcal{O}_{X}\left(x_{0}+\sigma\left(x_{0}\right)\right)$ is real; taking duals, varying $x_{0}$ and tensoring, there are then real line bundles on $X$ in any even degree. There are none in odd degree. In the same vein, one can show that quaternionic line bundles exist in degree $d$ if and only if $d-g+1 \equiv 0 \bmod 2[\mathrm{BHH}, \mathrm{AB}$, p. 55, Theorem 2.6]. For example, there is a quaternionic theta characteristic on $X$ if $g$ is even (see [At2, pp. 61-62]; recalling that $X_{\mathbb{R}}$ does not have any real point, there is no real theta characteristic on $X$ because there is no real line bundle on $X$ of odd degree).

Proposition 3.3. If the integer $d-g$ is even, then there is a real universal line bundle over $X \times \operatorname{Pic}^{d}(X)$.

If the degree $d$ is odd, then there is a quaternionic universal line bundle over $X \times \operatorname{Pic}^{d}(X)$.

Proof. Indeed, Lemma 3.1 and Lemma 3.2 allow us to produce all the universal bundles in the theorem from the real one in degree $g-2$, by twisting by an appropriate real or quaternionic line bundle. We note that there is a real line bundle on $X$ of degree two. Also, there is a quaternionic line bundle on $X$ of degree one (respectively, zero) if $g$ is even (respectively, odd) [BHH, Theorem 6.6] (see also [AB, p. 55, Theorem 2.6]).

3.2. Nonexistence: the case of even genus. First assume that the genus $g$ is an even integer.

Proposition 3.4. There is no real universal line bundle on $X \times \operatorname{Pic}^{2 d+1}(X)$.

There is no quaternionic universal line bundle on $X \times \operatorname{Pic}^{2 d}(X)$.

Proof. For any point $x \in X$, the line bundle $\mathcal{O}_{X}(x+\sigma(x))$ is real. Therefore, in view of Lemma 3.2. it suffices to prove the first part of the proposition for $d=0$.

Fix a quaternionic line bundle

$$
L_{0} \longrightarrow X
$$

such that degree $\left(L_{0}\right)=1$. Let

$$
\mathcal{L} \longrightarrow X \times \operatorname{Pic}^{0}(X)
$$

be a real universal line bundle; from Proposition 3.3 we know that such a universal line bundle exists. Let

$$
p_{X}: X \times \operatorname{Pic}^{1}(X) \longrightarrow X
$$


be the natural projection. We note that

$$
\mathcal{L}_{1}:=p_{X}^{*} L_{0} \otimes\left(\operatorname{Id}_{X} \times \varphi\right)^{*} \mathcal{L} \longrightarrow X \times \operatorname{Pic}^{1}(X)
$$

is a quaternionic universal line bundle.

To see that there is no real universal line bundle on $X \times \operatorname{Pic}^{1}(X)$, let

$$
p_{2}: X \times \operatorname{Pic}^{1}(X) \longrightarrow \operatorname{Pic}^{1}(X)
$$

be the natural projection. Any universal line bundle on $X \times \operatorname{Pic}^{1}(X)$ is of the form

$$
\mathcal{L}_{1} \otimes p_{2}^{*} \xi
$$

where $\mathcal{L}_{1}$ is the line bundle in (3.8). If the line bundle $\mathcal{L}_{1} \otimes p_{2}^{*} \xi$ in (3.9) is real, then $\xi$ must be a quaternionic line bundle, because $\mathcal{L}_{1}$ is quaternionic. On the other hand, it can be shown that there are no quaternionic line bundles on $\operatorname{Pic}^{1}(X)$. Indeed, the variety $\operatorname{Pic}^{1}\left(X_{\mathbb{R}}\right)$ defined over $\mathbb{R}$ has a real point because the line bundle $L_{0}$ in (3.7) is represented by a real point of $\operatorname{Pic}^{1}\left(X_{\mathbb{R}}\right)$; hence there is no quaternionic line bundle on $\operatorname{Pic}^{1}(X)$ (see CP p. 201, Proposition 2.7.4]).

For the second part, if one had a universal quaternionic bundle in even degree, one could use Lemma 3.2 to produce a universal real bundle in odd degree because there is a quaternionic line bundle on $X$ of degree one $\mathrm{BHH}$, Theorem 6.6]; we have just proven that these don't exist. This completes the proof of the proposition.

3.3. Nonexistence: the case of odd genus. Now assume that $g$ is odd.

Proposition 3.5. There is no real or quaternionic universal line bundle on $X \times$ $\operatorname{Pic}^{2 d}(X)$.

Proof. In view of Lemma 3.2 it suffices to prove the proposition for $d=0$.

Let

$$
\mathcal{L}^{\prime} \longrightarrow X \times \operatorname{Pic}^{0}(X)
$$

be a real or quaternionic universal line bundle. We will show that $\mathcal{L}^{\prime}$ can be modified to construct another real or quaternionic universal line bundle which is topologically trivial in the direction of $\mathrm{Pic}^{0}(X)$.

Let

$$
c \in H^{2}\left(\operatorname{Pic}^{0}(X), \mathbb{Z}\right)
$$

be the Künneth component of the Chern class $c_{1}\left(\mathcal{L}^{\prime}\right) \in H^{2}\left(X \times \operatorname{Pic}^{0}(X), \mathbb{Z}\right)$ of the line bundle in (3.10). Since $\mathcal{L}^{\prime}$ is real or quaternionic, the anti-holomorphic involution of $\operatorname{Pic}^{0}(X)$ (see (2.4) ) preserves $c$. Hence there is a real line bundle

$$
\xi \longrightarrow \operatorname{Pic}^{0}(X)
$$

such that $c_{1}(\xi)=c$ (see the paragraph following Remark 3.3.2 in [CP, p. 215]); note that the trivial line bundle $\mathcal{O}_{X}$ is fixed by the anti-holomorphic involution of $\mathrm{Pic}^{0}(X)$, so the assumption in $[\mathrm{CP}$. p. $214, \S 3.3]$ is fulfilled.

Let $p_{2}: X \times \operatorname{Pic}^{0}(X) \longrightarrow \operatorname{Pic}^{0}(X)$ be the natural projection. Consider the line bundle

$$
\mathcal{L}:=\mathcal{L}^{\prime} \otimes p_{2}^{*} \xi^{*} \longrightarrow X \times \operatorname{Pic}^{0}(X),
$$

where $\mathcal{L}^{\prime}$ and $\xi$ are constructed in (3.10) and (3.11), respectively. It is clear that $\mathcal{L}$ is real or quaternionic, and also it is topologically trivial in the direction of $\operatorname{Pic}^{0}(X)$.

Let

$$
A:=\operatorname{Pic}^{0}(X)^{\vee}
$$


be the moduli space of topologically trivial line bundles on $\operatorname{Pic}^{0}(X)$. The antiholomorphic involution $\widetilde{\sigma}$ of $\operatorname{Pic}^{0}(X)$ produces an anti-holomorphic involution of $A$ by sending any $\eta$ to $\widetilde{\sigma}^{*} \bar{\eta}$; this anti-holomorphic involution of $A$ will be denoted by $\sigma^{\prime}$.

We have the morphism

$$
f: X \longrightarrow A
$$

that sends any $x \in X$ to the point representing the restriction $\left.\mathcal{L}\right|_{\{x\} \times \operatorname{Pic}^{0}(X)}$, where $\mathcal{L}$ is constructed in (3.12). Note that $f$ is defined over $\mathbb{R}$, meaning $f(\sigma(x))=$ $\sigma^{\prime}(f(x))$ for all $x \in X$.

Fix a point $x_{0} \in X$. The divisor

$$
D=\frac{g-1}{2}\left(x_{0}+\sigma\left(x_{0}\right)\right)
$$

is real, meaning it is fixed by $\sigma$. We have an isomorphism

$$
\phi: \operatorname{Pic}^{0}(X) \longrightarrow \operatorname{Pic}^{g-1}(X)
$$

defined by $L \longmapsto L \otimes \mathcal{O}_{X}(D)$. This morphism intertwines the anti-holomorphic involutions of $\operatorname{Pic}^{0}(X)$ and $\operatorname{Pic}^{g-1}(X)$, so it is defined over $\mathbb{R}$. Let

$$
\Theta_{0}:=\phi^{-1}(\Theta) \subset \operatorname{Pic}^{0}(X)
$$

be the inverse image of the theta divisor on $\operatorname{Pic}^{g-1}(X)$ (see the proof of Lemma 3.1 for the definition of a theta divisor).

For any $L \in \operatorname{Pic}^{0}(X)$, let

$$
\tau_{L}: \operatorname{Pic}^{0}(X) \longrightarrow \operatorname{Pic}^{0}(X)
$$

be the isomorphism defined by $L^{\prime} \longmapsto L^{\prime} \otimes L$. The divisor $\Theta_{0}$ in 3.15) produces an isomorphism

$$
\operatorname{Pic}^{0}(X) \stackrel{\sim}{\longrightarrow} A
$$

(see (3.13) for $A$ ) by sending any $L$ to the line bundle $\mathcal{O}_{\operatorname{Pic}^{0}(X)}\left(\tau_{L}^{*} \Theta_{0}-\Theta_{0}\right)$; this map is an isomorphism because $\Theta_{0}$ gives a principal polarization on $\operatorname{Pic}^{0}(X)$. Let

$$
\Theta^{\prime} \subset A
$$

be the image of $\Theta_{0}$ by the isomorphism in (3.16).

Note that the pullback $f^{*} \mathcal{O}_{A}\left(\Theta^{\prime}\right) \longrightarrow X$ is a real line bundle, where $f$ is the morphism in (3.14). We have

$$
\operatorname{degree}\left(f^{*} \mathcal{O}_{A}\left(\Theta^{\prime}\right)\right)=g
$$

(see [GH, p. 336]). We note that (3.17) also follows from (3.3) and (3.4), because they imply that degree $\left(\left.\phi^{*} \mathcal{O}_{\mathrm{Pic}^{g-1}(X)}(\Theta)\right|_{X \times\{\zeta\}}\right)=\operatorname{degree}\left(K_{X}\right)-\operatorname{degree}(\zeta)=g$.

On the other hand, since $g$ is odd, there is no real line bundle on $X$ of degree $g$ (recall that $\sigma$ does not have any fixed point). This completes the proof of the proposition. 


\section{REAL UNIVERSAL BUNDLES OF HIGHER RANK}

4.1. Existence of universal bundles. Henceforth, we will assume that

(1) the rank $r$ is at least two, and

(2) $d$ is coprime to $r$.

Recall the construction of the universal bundle in Lemma 2.1.

Remark 4.1. The integer $a$ in (2.8) can be taken to be even if and only if the integer $\chi$ in (2.7) is odd. Indeed, if $\chi$ is even, then $r$ is odd (recall that $d$ is coprime to $r$ ), and hence from (2.8) we know that $a r$ is odd. Therefore, $a$ is odd if $\chi$ is even. If $\chi$ is odd, then $\chi$ is coprime to $2 r$. Hence there are integers $a^{\prime}$ and $b$ such that $2 r a^{\prime}+\chi b=-1$. Hence $a$ can be taken to be even if $\chi$ is odd.

Proposition 4.2. If the integer $\chi$ in (2.7) is odd, then there is a real universal vector bundle over $X \times \mathcal{M}_{X}(r, d)$.

Thus there are real universal bundles when:

- $g$ even: the degree and the rank are of opposite parity.

- $g$ odd: the degree is odd.

Given a real (respectively, quaternionic) line bundle on $X$ of degree $d_{0}$, then there is a universal real bundle over $X \times \mathcal{M}_{X}(r, d)$ if and only if there is a universal real (respectively, quaternionic) bundle over $X \times \mathcal{M}_{X}\left(r, d+r d_{0}\right)$.

In particular, for $d$ odd, there is a universal quaternionic vector bundle over $X \times \mathcal{M}_{X}(r, d)$.

Proof. In view of Remark 4.1, the first part is a consequence of Lemma 2.1. The second part follows as in Lemma 3.2. For the third, let us consider first the case when the genus is odd. There is a quaternionic line bundle of degree zero on $X$. The second part of the proposition then tells us that there is a universal quaternionic bundle if and only if there is a real one in a given rank and degree; thus the quaternionic bundle exists for odd degree. For even genus, one has a quaternionic line bundle of degree one. One then has a universal quaternionic bundle over $X \times \mathcal{M}_{X}(r, d)$ if one has a universal real bundle over $X \times \mathcal{M}_{X}(r, d-r)$. This happens when $r, d-r$ are of opposite parity, that is, when $d$ is odd.

4.2. Nonexistence: the real case of even degree. Assume that the degree $d$ is even.

Proposition 4.3. There is a real universal vector bundle over $X \times \mathcal{M}_{X}(r, d)$ if and only if $\chi=d-r(g-1)$ is odd, i.e., if and only if $r$ is odd and $g$ is even.

Proof. First note that the rank $r$ is odd because $d$ is coprime to $r$. So, if $g$ is even, then $\chi$ is odd. Hence, in that case, from Proposition 4.2 it follows that there is a real universal vector bundle over $X \times \mathcal{M}_{X}(r, d)$.

Assume that $g$ is odd.

We will construct a real cyclic étale cover

$$
f: \tilde{X} \longrightarrow X
$$

of degree $r$. For that, first note that there is a real line bundle over $X$ of order exactly $r$. Indeed, if $\widetilde{\sigma}$ is the anti-holomorphic involution of $\operatorname{Pic}^{0}(X)$ defined by $\eta \longmapsto \sigma^{*} \bar{\eta}$, then the connected component of the fixed point locus $\operatorname{Pic}^{0}(X)^{\widetilde{\sigma}}$ containing the trivial line bundle $\mathcal{O}_{X}$ is a divisible group. Also, all line bundles lying 
in this component are real because $\mathcal{O}_{X}$ is a real line bundle. Take any point $L_{0}$ of order exactly $r$ of this component. So $L_{0}$ is a real line bundle of order exactly $r$. Now consider the map of total spaces of line bundles

$$
\rho: L_{0} \longrightarrow L_{0}^{\otimes r}=\mathcal{O}_{X}
$$

defined by $v \longmapsto v^{\otimes r}$. Take

$$
\widetilde{X}=\rho^{-1}\left(1_{X}\right),
$$

where $1_{X}$ is the image of the section of $\mathcal{O}_{X}$ defined by the constant function 1 . The projection $f$ in (4.1) is the restriction of the natural projection of $L_{0}$ to $X$.

The anti-holomorphic involution of the total space of the line bundle $L_{0}$ defining its real structure preserves the subset $\widetilde{X}$. Therefore, this restriction produces an anti-holomorphic involution $\sigma_{\widetilde{X}}$ of $\widetilde{X}$. Note that the projection $f$ intertwines $\sigma_{\widetilde{X}}$ and $\sigma$.

We have

$$
\widetilde{g}:=\operatorname{genus}(\widetilde{X})=r(g-1)+1,
$$

and $\widetilde{g}$ is odd because $g$ is odd.

Since $d$ is even and $\widetilde{g}$ is odd, there is a quaternionic line bundle

$$
\xi \longrightarrow \widetilde{X}
$$

of degree $d[\mathrm{BHH}$, Theorem 6.6] (see also [AB, p. 55, Theorem 2.6]). Consider the direct image

$$
E:=f_{*} \xi \in \mathcal{M}_{X}(r, d),
$$

where $f$ is the projection in (4.1); since $f$ is unramified, the vector bundle $f_{*} \xi$ is semistable of degree $d$; hence it is stable (recall that $d$ is coprime to $r$ ).

Since $\xi$ is quaternionic, it follows that $E$ is quaternionic; the direct image of the isomorphism

$$
\xi \longrightarrow \sigma_{\widetilde{X}}^{*} \bar{\xi}
$$

defining the quaternionic structure of $\xi$ is clearly a quaternionic structure on $E$. Note that $E$ is a real point of $\mathcal{M}_{X}(r, d)$, meaning that it is fixed by the antiholomorphic involution of $\mathcal{M}_{X}(r, d)$.

If there is a real universal vector bundle $\mathcal{E}$ over $X \times \mathcal{M}_{X}(r, d)$, then the restriction of $\mathcal{E}$ to $X \times\{E\}=X$ is also real. Consequently, in that case, $E$ would be both real and quaternionic. But that's impossible $[\mathrm{BHH}]$. Hence there is no universal real algebraic vector bundle over $X \times \mathcal{M}_{X}(r, d)$. This completes the proof of the proposition.

4.3. Nonexistence: the real case of odd degree. We now assume that $d$ is odd.

Proposition 4.4. There is a real universal vector bundle over $X \times \mathcal{M}_{X}(r, d)$ if and only if $\chi=d-r(g-1)$ is odd, i.e., if and only if either $r$ is even or $g$ is odd.

Proof. The cases for which the bundles must exist have been covered above; one must show nonexistence in the case when $r$ is odd, $g$ is even.

Fix a real cyclic étale cover

$$
f: \tilde{X} \longrightarrow X
$$


of degree $r$ (see (4.1) for its construction). Note that

$$
\widetilde{g}:=\operatorname{genus}(\widetilde{X})=1+(g-1) r
$$

is even.

Let

$$
\xi \longrightarrow \tilde{X}
$$

be a real line bundle of degree $d-r$ (note that $d-r$ is even). The vector bundle

$$
E:=f_{*} \xi \longrightarrow X
$$

is stable of rank $r$ and degree $d-r$. The real structure of $\xi$ defines a real structure on $E$. Let

$$
\varphi: \operatorname{Pic}^{1}(X) \longrightarrow \mathcal{M}_{X}(r, d)
$$

be the morphism defined by $L \longmapsto E \otimes L$. Since the vector bundle $E$ is real, the morphism $\varphi$ is defined over $\mathbb{R}$, meaning $\varphi$ intertwines the anti-holomorphic involutions of $\operatorname{Pic}^{1}(X)$ and $\mathcal{M}_{X}(r, d)$.

To prove by contradiction, let

$$
\mathcal{U} \longrightarrow X \times \mathcal{M}_{X}(r, d)
$$

be a real universal vector bundle. Let

$$
\mathcal{P}:=\left(\operatorname{Id}_{X} \times \varphi\right)^{*} \mathcal{U} \longrightarrow X \times \operatorname{Pic}^{1}(X)
$$

be the pullback, where $\varphi$ is the map in (4.6). Let

$$
\mathcal{L}:=\left(\bigwedge^{r} \mathcal{P}\right) \otimes p_{X}^{*} \bigwedge^{r} E^{*} \longrightarrow X \times \operatorname{Pic}^{1}(X)
$$

be the line bundle, where $p_{X}$ is the projection of $X \times \operatorname{Pic}^{1}(X)$ to $X$. Since both the vector bundles $\mathcal{P}$ and $E$ are real, it follows that the line bundle $\mathcal{L}$ is also real.

There is a quaternionic universal line bundle

$$
\mathcal{L}_{0} \longrightarrow X \times \operatorname{Pic}^{1}(X)
$$

(see Proposition 3.4). Since $r$ is odd, the line bundle $\mathcal{L}_{0}^{\otimes r}$ is also quaternionic. Note that for any $L \in \operatorname{Pic}^{1}(X)$, the restrictions of both the line bundles $\mathcal{L}$ and $\mathcal{L}_{0}^{\otimes r}$ to $X \times\{L\}$ are isomorphic to $L^{\otimes r}$. Hence there is a unique, up to an isomorphism, holomorphic line bundle

$$
L_{1} \longrightarrow \operatorname{Pic}^{1}(X)
$$

such that

$$
\mathcal{L}_{0}^{\otimes r}=\mathcal{L} \otimes p_{2}^{*} L_{1},
$$

where $p_{2}$ is the projection of $X \times \operatorname{Pic}^{1}(X)$ to $\operatorname{Pic}^{1}(X)$. Since $\mathcal{L}$ is real and $\mathcal{L}_{0}^{\otimes r}$ is quaternionic, we conclude that $L_{1}$ is quaternionic.

Since $g$ is even, there is a quaternionic line bundle on $X$ of degree one; this follows from the facts that there is a quaternionic theta characteristic on $X$, At2, pp. 61-62], and that there is a real line bundle on $X$ of degree two (see the proof of Proposition 3.4). Hence $\operatorname{Pic}^{1}(X)$ has a real point. Therefore, there is no quaternionic line bundle on $\operatorname{Pic}^{1}(X)$ [CP, p. 201, Proposition 2.7.4]. This contradicts the existence of $L_{1}$ in (4.8). Therefore, there is no real universal vector bundle over $X \times \mathcal{M}_{X}(r, d)$. This completes the proof of the proposition. 


\subsection{Nonexistence: the quaternionic case.}

Proposition 4.5. There is a universal quaternionic vector bundle over the Cartesian product $X \times \mathcal{M}_{X}(r, d)$ if and only if $d$ is odd.

The proof proceeds by using the results for the real case, tensoring by a quaternionic line bundle of degree 0 (if $g$ is odd) or 1 (if $g$ is even); universal real vector bundles exist if and only if the corresponding real ones exist.

\section{FiXed DETERMINANT MODULI SPACE}

As before, assume that $r \geq 2$.

Let $\zeta \longrightarrow X$ be a holomorphic line bundle of degree $d$. Let

$$
\mathcal{M}_{X}(r, \zeta) \subset \mathcal{M}_{X}(r, d)
$$

be the moduli space of stable vector bundles $E$ with $\bigwedge^{r} E=\zeta$. There is a universal vector bundle over $X \times \mathcal{M}_{X}(r, \zeta)$ if and only if $d$ is coprime to $r$ [Ra].

As in Section 4, assume that $d$ is coprime to $r$.

Let $\zeta$ be a fixed point of the anti-holomorphic involution $\widetilde{\sigma}$ of $\operatorname{Pic}^{d}(X)$ (see (2.4)). So the line bundle $\zeta \longrightarrow X$ is either real or quaternionic. The subvariety $\mathcal{M}_{X}(r, \zeta)$ is preserved by the anti-holomorphic involution of $\mathcal{M}_{X}(r, d)$ (see (2.6) ). The induced anti-holomorphic involution of $\mathcal{M}_{X}(r, \zeta)$ will be denoted by $\widehat{\sigma}$.

A real universal vector bundle over $X \times \mathcal{M}_{X}(r, \zeta)$ is a universal vector bundle

$$
\mathcal{E} \longrightarrow X \times \mathcal{M}_{X}(r, \zeta)
$$

equipped with a holomorphic isomorphism

$$
\sigma^{\prime}: \mathcal{E} \longrightarrow(\sigma \times \widehat{\sigma})^{*} \overline{\mathcal{E}}
$$

such that $\sigma^{\prime} \circ \sigma^{\prime}=\operatorname{Id}_{\mathcal{E}}$.

Therefore, the restriction of a real universal vector bundle over $X \times \mathcal{M}_{X}(r, d)$ to $X \times \mathcal{M}_{X}(r, \zeta)$ is also real universal.

Proposition 5.1. Assume that the line bundle $\zeta$ is real. There is a real universal vector bundle over $X \times \mathcal{M}_{X}(r, \zeta)$ if and only if $\chi(E)$ is odd for $E \in \mathcal{M}_{X}(r, \zeta)$.

Proof. Since $\zeta$ is real, the degree $d$ is even. So $r$ is odd. In view of Proposition 4.3 , the only case to check is where $r$ and $g$ are both odd.

We can choose the line bundle $\xi \longrightarrow \widetilde{X}$ in (4.2) such that the vector bundle $f_{*} \xi$ in (4.3) lies in $\mathcal{M}_{X}(r, \zeta)$. Indeed, this follows from the fact that the connected component of the fixed point locus $\operatorname{Pic}^{0}(X)^{\widetilde{\sigma}}$ containing $\mathcal{O}_{X}$ is a divisible group. Hence the argument in Proposition 4.3 completes the proof for the case where both $r$ and $g$ are odd.

Proposition 5.2. Assume that the line bundle $\zeta$ is quaternionic. There is a real universal vector bundle over $X \times \mathcal{M}_{X}(r, \zeta)$ if and only if there is a real universal vector bundle over $X \times \mathcal{M}_{X}(r, d)$.

Proof. First assume that $d$ is even. As in Proposition 5.1, the only case to check is the one where both $r$ and $g$ are odd. Again the argument in Proposition 4.3 settles this case.

Now assume that $d$ is odd.

In view of Proposition 4.4 the only case to check is the one where $r$ is odd and $g$ is even. 
Consider the covering $\widetilde{X}$ in (4.4). Its genus $\widetilde{g}$ is even (see (4.5)). Hence there is a quaternionic line bundle

$$
L \longrightarrow \tilde{X}
$$

such that $f_{*} L \in \mathcal{M}_{X}(r, \zeta)$, where $f$ and $\tilde{X}$ are as in (4.4). See the proof of Proposition 3.4 for the existence of $L$; we note that the determinant can always be arranged to be $\zeta$ because the connected component of $\operatorname{Pic}^{0}(X)^{\widetilde{\sigma}}$ containing $\mathcal{O}_{X}$ is a divisible group.

The vector bundle $f_{*} L$ is quaternionic because $L$ is quaternionic. In particular, the point of $\mathcal{M}_{X}(r, \zeta)$ representing $f_{*} L$ is fixed by the anti-holomorphic involution of $\mathcal{M}_{X}(r, \zeta)$.

If

$$
\mathcal{U} \longrightarrow X \times \mathcal{M}_{X}(r, \zeta)
$$

is a real universal vector bundle, then the restriction of $\mathcal{U}$ to $X \times\left\{f_{*} L\right\}$ is real. But we have seen that $f_{*} L$ is quaternionic. This is a contradiction [BHH]. Hence there is no real universal vector bundle over $X \times \mathcal{M}_{X}(r, \zeta)$ if $r$ is odd and $g$ is even. This completes the proof of the proposition.

\section{ACKNOWLEDGEMENTS}

The first-named author would like to thank McGill University for its hospitality.

\section{REFERENCES}

[ACGH] E. Arbarello, M. Cornalba, P. A. Griffiths and J. Harris: Geometry of algebraic curves. Vol. I, Springer-Verlag, New York, 1985. MR770932 (86h:14019)

[AB] P. Arés-Gastesi and I. Biswas: The Jacobian of a nonorientable Klein surface, II, Proc. Indian Acad. Sci. (Math. Sci.) 113 (2003), 139-152. MR1983747(2004g:30061)

[At1] M. F. Atiyah: Vector bundles over an elliptic curve, Proc. London Math. Soc. 7 (1957), 412-452. MR0131423 (24:A1274)

[At2] M. F. Atiyah: Riemann surfaces and spin structures, Ann. Sci. École Norm. Sup. 4 (1971), 47-62. MR0286136 (44:3350)

[BM] A. Beilinson and Y. I. Manin: The Mumford form and the Polyakov measure in string theory, Comm. Math. Phys. 107 (1986), 359-376. MR866195(88a:32035)

$[\mathrm{BHH}] \quad$ I. Biswas, J. Huisman and J. Hurtubise: The moduli space of stable vector bundles over a real algebraic curve, Math. Ann. 347 (2010), 201-233. MR2593289

[CP] C. Ciliberto and C. Pedrini: Real abelian varieties and real algebraic curves, in: Lectures in real geometry (Madrid, 1994), 167-256, de Gruyter Exp. Math., 23, de Gruyter, Berlin, 1996. MR1440212 (99a:14080)

[De] P. Deligne: Le déterminant de la cohomologie, Current trends in arithmetical algebraic geometry (Arcata, Calif., 1985), 93-177, Contemp. Math., 67, Amer. Math. Soc., Providence, RI, 1987. MR.902592 (89b:32038)

[GH] P. A. Griffiths and J. Harris: Principles of algebraic geometry, Pure and Applied Mathematics, Wiley-Interscience, New York, 1978. MR.507725 (80b:14001)

[Gr] A. Grothendieck: Sur la classification des fibrés holomorphes sur la sphère de Riemann, Amer. Jour. Math. 79 (1957), 121-138. MR0087176(19:315b)

[HJ] N.-K. Ho and L. C. Jeffrey, The volume of the moduli space of flat connections on a nonorientable 2-manifold, Comm. Math. Phys. 256 (2005), 539-564. MR 2327950 (2008e:53171)

[HL] N.-K. Ho and C.-C. M. Liu: Yang-Mills connections on nonorientable surfaces, Comm. Anal. Geom. 16 (2008), 617-679. MR2429971(2009k:53054)

[JK] L. C. Jeffrey and F. C. Kirwan: Intersection theory on moduli spaces of holomorphic bundles of arbitrary rank on a Riemann surface, Ann. of Math. (2) 148 (1998), 109-196. MR 1652987 (2000c:14045)

$[\mathrm{Mu}] \quad$ D. Mumford: Geometric invariant theory, Springer-Verlag, 1965. MR 0214602 (35:5451) 
[MN] D. Mumford and P. E. Newstead: Periods of a moduli space of bundles on curves, Amer. Jour. Math. 90 (1968), 1200-1208. MR0234958 (38:3272)

[Ne] P. E. Newstead: A non-existence theorem for families of stable bundles, Jour. London Math. Soc. 6 (1973), 259-266. MR0311662(47:224)

[Ra] S. Ramanan: The moduli spaces of vector bundles over an algebraic curve, Math. Ann. 200 (1973), 69-84. MR0325615(48:3962)

School of Mathematics, Tata Institute of Fundamental Research, Homi Bhabha ROAD, BOMBAy 400005, INDIA

E-mail address: indranil@math.tifr.res.in

Department of Mathematics, McGill University, Burnside Hall, 805 Sherbrooke Street W., Montreal, Québec, Canada H3A 2K6

E-mail address: jacques.hurtubise@mcgill.ca 